\title{
PERTURBATIONS OF NORMALLY SOLVABLE NONLINEAR OPERATORS, I
}

\section{WILLIAM O. RAY and ANITA M. WALKER}

\author{
Department of Mathematics \\ The University of Oklahoma \\ Norman, OK 73019 \\ (Received January 16, 1984)
}

ABSTRACT. Let $X$ and $Y$ be Banach spaces and let $\mathcal{F}$ and $\boldsymbol{G}$ be Gateaux differentiable mappings from $X$ to $Y$. In this note we study when the operator $\mathcal{F}+\boldsymbol{G}$ is surjective for sufficiently small perturbations $\boldsymbol{G}$ of a surjective operator $F$. The methods extend previous results in the area of normal solvability for nonlinear operators.

KEY WORDS AND PHRASES. Solvability, normal solvability, inverse function theorems,

Ekeland's Theorem, contractor directions. AMS/MOS Subject classification. $47 \mathrm{H15}$.

Let $F$ be a nonlinear mapping from a Banach space $X$ to a Banach space $Y$. We call $F$ Gateaux differentiable if for each $x \in X$ there is an operator $\mathcal{F}^{\prime}(x)$, not necessarily linear, mapping $X$ to $Y$ for which

$$
\lim _{t \rightarrow 0} \frac{F(x+t h)-F(x)}{t}=F^{\prime}(x)(h)
$$

for all $h \in X$. This operator $F$ is normally solvable if the injectivity of the set of adjoint operators $F^{\prime}(x) *$ implies surjectivity of $F$.

In this light, the theory of normally solvable operators has been investigated by, among others, S.I. Phohzhayev [1], F.E. Browder [2], [3], [4] and [5], W.A. Kirk and J. Caristi [6], D. Downing and W.A. Kirk [7], M. Altman [8], W.J. Cramer and W.O. Ray [9] and J. Kolomy [10]. Throughout these works the injectivity of each of the operators $\mathcal{F}(x) *$ has been implicit assumption. If it is assumed also that each $F^{\prime}(x)$ * has a bounded inverse, then similar surjectivity results are obtained by weakening an assumption on the closure (or weak enclosure) of $\mathcal{F}(\mathrm{X})$. When $F^{\prime}(x)$ * has a bounded inverse, Newton's method can be employed to solve the equation $F(x)=0$; if the bounded inverse assumption is removed, J. Moser [11] showed that Newton's method can be modified to still yield a solution of $\boldsymbol{F}(\mathbf{x})=0$. (See also W.0. Ray [12]).

In this note we avoid the explicit use of adjoint operators; instead we consider a uniformly surjective Gateaux differentiable operator $\mathcal{F}$ which we perturb by a "small" Gateaux differentiable operator $\boldsymbol{G}$. Under the assumptions we make on $G^{\prime}(x)$ we demonstrate, via a transfinite Newton's method, the surjectivity of the operator $\mathfrak{F}+\mathfrak{G}$. Hence Newton's method is sufficiently regular that small 
perturbations of a differentiable operator do not hinder the convergence of the iterates.

As the primary tool in deriving our main result, we make use of an extension of Caristi's Theorem formulated in [13]:

THEOREM 1. Let $(M, d)$ be a complete metric space and let $\varphi: M \rightarrow[0, \infty)$ be a lower semicontinuous function. Let $c:[0, \infty) \rightarrow(0, \infty)$ be a continuous nonincreasing function for which $\int^{\infty} c(u) d u=\infty$, and let $x_{0} \in M$ be fixed. If $g$ is a mapping of $M$ into itself which satisfies

$$
c\left(d\left(x, x_{0}\right)\right) d(x, g(x)) \leq \varphi(x)-\varphi(g(x)) \quad(x \in M),
$$

then $g$ has a fixed point in $M$.

Our main result is the following theorem, the proof of which is similar to those found in the earlier paper [13].

THEOREM 2. Let $X$ and $Y$ be Banach spaces and let $\mathcal{F}$ and $\boldsymbol{G}$ be Gateaux differentiable mappings from $X$ to $Y$. Let $c:[0, \infty) \rightarrow(0, \infty)$ be a continuous non-increasing function for which $\int^{\infty} c(u) d u=\infty$. Suppose for each $x \in X$ that

$$
\begin{aligned}
& G^{\prime}(x) \text { is a bounded linear operator from } X \text { to } Y, \\
& \text { and } \\
& F^{\prime}(x)(B(0 ; 1)) \supseteq B(0 ; C(\|x\|)) .
\end{aligned}
$$

Suppose, in addition, for some $\mu \in(0,1)$ and each $x \in X$ that

$$
c(\|x\|)^{-1}\left\|G^{\prime}(x)\right\| \leq \mu \text {. }
$$

If the mapping $\mathfrak{P} \equiv \mathcal{F}+\mathfrak{G}$ has closed graph, then $\mathfrak{P}$ is an open mapping from $X$ onto $\mathrm{Y}$.

(Note that if $F^{\prime}(x)$ is linear, invertible and if $\left\|F^{\prime}(x)^{-1}\right\| \leq c(\|x\|)$, then (2) is automatically fulfilled.) As a simple consequence it is enough to assume that $\mathcal{F}$ and $\boldsymbol{G}$ each have closed graph.

THEOREM 3. Let $\mathcal{F}$ and $\boldsymbol{G}$ be given as in Theorem 2. Define the Gateaux differentiable operator $\mathcal{P}: X \rightarrow Y$ by $\mathcal{P}=\mathcal{F}+\boldsymbol{G}$. If $\mathcal{F}$ and $\boldsymbol{G}$ have closed graph, then so does $P$; in particular, $P$ is an open mapping from $X$ onto $Y$.

The proof that $\mathbf{P}$ is open in Theorems 2 and 3 follows readily from a direct application of Theorem 2.1 of [9]. Instrumental in verifying both the surjectivity and openness conclusions above are a pair of "contractor inequalities" (cf. [8]). These inequalities are given by:

LEMMA. Let $\mathcal{F}$ and $\mathcal{G}$ be given as in Theorem 2. Let $\mathcal{P}: X \rightarrow Y$ be defined by $\mathbf{P}=\mathcal{F}+\boldsymbol{G}$. Then there is a $q \in(\mu, 1)$ such that for each $y \in Y$ there is a $t \in(0,1]$ and an $\bar{x} \in X$ such that

$$
\text { and } \begin{aligned}
& \left\|\mathbf{p}_{\bar{x}}-\mathbf{P}_{\mathrm{x}}-\mathrm{t}\left(\mathrm{y}-\mathbf{p}_{\mathrm{x}}\right)\right\| \leq \mathrm{qt}\left\|\mathrm{y}-\mathbf{P}_{\mathrm{x}}\right\|, \\
& \|\overline{\mathrm{x}}-\mathrm{x}\|) \leq \mathrm{c}(\|\mathrm{x}\|)^{-1} t\left\|\mathrm{y}-\mathbf{p}_{\mathrm{x}}\right\| .
\end{aligned}
$$

Similar inequalities can be deduced from injectivity hypotheses on $\mathbf{p}^{\prime}(\mathbf{x})$ * (see the survey [14] for a discussion). It is noteworthy that the uniform surjectivity hypothesis (2) is somewhat weaker than the corresponding assumptions on the adjoint $P^{\prime}(x)$ * 
When specialized to the case that the function $c(u)$ is a constant, the above extends a result of I. Rosenholtz and W.0. Ray [15].

THEOREM 4. Let $X$ and $Y$ be Banach spaces and let $F$ and $\boldsymbol{G}$ be Gateaux differentiable mappings from $X$ to $Y$. Suppose for each $x \in X$ that

$$
\begin{aligned}
& G^{\prime}(x) \quad \text { is a bounded and linear operator from } X \text { to } Y \text {, } \\
& \text { and } \\
& F^{\prime}(x)(B(0 ; 1)) \supseteq B(0 ; \delta) \text { for some } \delta>0 .
\end{aligned}
$$

Suppose, in addition, for some $\mu \in(0,1)$ that

$$
\delta^{-1}\left\|G^{\prime}(x)\right\| \leq \mu \text {. }
$$

If the operator $\mathbb{P}=\boldsymbol{F}+\boldsymbol{G}$ has closed graph, then $\mathfrak{P}$ is an open surjection. In particular, if $\mathcal{F}$ and $\mathcal{G}$ each have closed graph, then $\mathcal{P}$ is an open surjection.

We now present the proofs of the above theorems; we first verify the Lemma and then use it to prove our main results.

PROOF OF LEMMA. Fix $y \in Y$ and $q \in(\mu, 1)$. If $y=P(x)$ for some $x$, then choose $\bar{x}=x$ and the conclusions follow for any $t$.

So without loss of generality we may assume $y \notin P(X)$. For each $x \in X$, $c(\|x\|)\|y-P x\|^{-1}(y-P x) \in B(0 ; c(\|x\|))$. By (2) there exists a $w \in B(0 ; 1)$ so that $F^{\prime}(x)(w)=c(\|x\|)\left\|y-P_{x}\right\|^{-1}\left(y-P_{x}\right)$. Set $h=c(\|x\|)^{-1}\left\|y-P_{x}\right\| w$. Then the homogeneity of $\mathcal{F}^{\prime}(\mathrm{x})$ implies that $\mathcal{F}^{\prime}(\mathbf{x})(\mathrm{h})=\mathrm{y}-\mathbf{P x}_{\mathbf{x}}$, with $\|\mathrm{h}\| \leq \mathrm{c}(\|\mathbf{x}\|)^{-1} \| \mathrm{y}-\mathbf{P x}_{\mathbf{x}}$.

By hypothesis both $\mathcal{F}$ and $\mathcal{G}$ are Gateaux differentiable, so we can choose $t \in(0,1]$ so small that

$$
\begin{aligned}
& \left\|F(x+t h)-F(x)-t F^{\prime}(x)(h)\right\| \\
& \quad \leq \frac{1}{2}(q-\mu) t\|y-P x\|,
\end{aligned}
$$

and

$$
\begin{aligned}
& \left\|G(x+t h)-G(x)-t G^{\prime}(x)(h)\right\| \\
& \quad \leq \frac{1}{2}(q-\mu) t\|y-P(x)\| .
\end{aligned}
$$

Setting $\bar{x}=x+t h$ and combining (9) and (10) yields, via the triangle and (3), that

$$
\begin{aligned}
& \left\|P_{\bar{x}}-P_{x}-t(y-x)\right\| \leq\left\|F \bar{x}-F_{x}-t^{\prime} F^{\prime}(x)(h)\right\|+\left\|G \bar{x}-G x-t G^{\prime}(x)(h)\right\| \\
& +t\left\|F^{\prime}(x)(h)-(y-P x)\right\|+t\left\|G^{\prime}(x)(h)\right\| \\
& \leq \frac{1}{2}(q-\mu) t\|y-p x\|+\frac{1}{2}(q-\mu) t\|y-P x\| \\
& +0+t\left\|G^{\prime}(x)\right\| \cdot\|h\| \\
& \leq(q-\mu) t\|y-p x\|+t \cdot c(\|x\|) \mu c(\|x\|)^{-1}\|y-p x\| \\
& =\mathrm{qt}\|\mathrm{y}-\mathrm{px}\| \text {, }
\end{aligned}
$$

giving (4).

To derive (5) observe that $\|\bar{x}-\mathbf{x}\|=t\|h\| \leq c(\|x\|)^{-1} t\|y-p x\|$. We now prove Theorem 2 .

PROOF. We begin by demonstrating the surjectivity of $\mathbf{P}=\boldsymbol{F}+\boldsymbol{G}$.

Define a metric $p$ on $x$ by $p(x, y)=\max \left\{(1+q)\|x-y\|, c(0)^{-1}\left\|p_{x}-p y\right\|\right\}$.

Since $P$ has closed graph, $(x, p)$ is a complete metric space.

Fix $y \in Y$ and $q \in(\mu, 1)$. Set $\varphi(x)=(1+q)(1-q)^{-1}\|y-p x\|$. Then $\varphi$ is continuous from $(X, p)$ to $[0, \infty)$ since $p$ has closed graph. 
We proceed by contradiction, so suppose $y \notin \mathcal{P}(x)$. Then by the Lemma, defining $g: X \rightarrow X$ by $g(x)=\bar{x}$, it follows that

$$
\text { and } \begin{aligned}
& \left\|P(g(x))-P_{x}-t\left(y-P_{x}\right)\right\| \leq q t\left\|y-P_{x}\right\|, \\
& c(\|x\|)\|g(x)-x\| \leq t\left\|y-P_{x}\right\| .
\end{aligned}
$$

Note that since $y \neq \mathcal{P}_{x}$, it follows that $g(x) \neq x$ for each $x \in X$. Applying the triangle inequality to (11) gives

$$
\left\|\mathbf{P}(\mathrm{g}(\mathrm{x}))-\mathbf{P}_{\mathbf{x}}\right\| \leq(1+q) t\left\|\mathbf{y}-\mathbf{P}_{\mathbf{x}}\right\| .
$$

A second application of the triangle inequality gives

$$
\begin{aligned}
& \|\mathbf{p}(g(x))-y\|-(1-t)\left\|\mathbf{p}_{x}-y\right\| \leq q t\left\|y-p_{x}\right\|, \\
& t\left\|y-p_{x}\right\| \leq(1-q)^{-1}\left(\left\|p_{x}-y\right\|-\|p(g(x))-y\|\right) .
\end{aligned}
$$

Now, (13) and (14) together imply

$$
\begin{aligned}
\|\mathfrak{P}(\mathrm{g}(\mathrm{x}))-\mathfrak{p} \mathbf{x}\| & \leq(1+q)(1-q)^{-1}\left(\left\|\mathfrak{p}_{\mathbf{x}}-\mathrm{y}\right\|-\|\mathfrak{P}(\mathrm{g}(\mathrm{x}))-\mathrm{y}\|\right) \\
& =\varphi(\mathbf{x})-\varphi(\mathrm{g}(\mathrm{x})) .
\end{aligned}
$$

Also (12) and (14) imply

$$
\begin{aligned}
& (1+q) c(\|x\|)\|g(x)-x\| \\
& \quad \leq(1+q)(1-q)^{-1} \times\left(\left\|\mathbf{p}_{x}-y\right\|-\|P(g(x))-y\|\right) \\
& \quad=\varphi(x)-\varphi(g(x)) .
\end{aligned}
$$

Now let $x_{0}=0$ be fixed in $x$. Consider first if $p(x, 0)=(1+q)\|x\|$. Then $\|x\| \leq(1+q)\|x\|$ implies that $c((1+q)\|x\|) \leq c(\|x\|)$ since $c$ is nonincreasing. Likewise, if $p(x, 0)=c(0)^{-1}\left\|\mathbf{p}_{x}-\mathbf{p}(0)\right\|$, (and thus $\left.(1+q)\|x\| \leq c(0)^{-1}\left\|\mathbf{p x}_{\mathbf{x}}-\mathbf{p}(0)\right\|\right)$, then $\|\mathbf{x}\| \leq c(0)^{-1}\left\|\mathbf{p x}_{\mathbf{x}}-\mathbf{P}(0)\right\|$; so $c\left(c(0)^{-1}\|\mathbf{p x}-\mathbf{P}(0)\|\right) \leq c(\|\mathbf{x}\|)$. Hence in either case, $c(p(x, 0)) \leq c(\|x\|)$.

Now, if $\mathrm{p}(\mathrm{x}, \mathrm{g}(\mathrm{x}))=(1+q)\|\mathrm{x}-\mathrm{g}(\mathrm{x})\|$, then (16) implies

$$
\begin{aligned}
c(p(x, 0)) p(x, g(x)) & \leq c(\|x\|)(1+q)\|x-g(x)\| \\
& \leq \varphi(x)-\varphi(g(x)),
\end{aligned}
$$

so (*) holds, while if $\mathrm{p}(\mathrm{x}, \mathrm{g}(\mathrm{x}))=\mathrm{c}(0)^{-1}\left\|\mathrm{px}_{\mathrm{x}}-\mathrm{p}(\mathrm{g}(\mathrm{x}))\right\|$, then (15) Implies

$$
\begin{aligned}
c(p(x, 0))_{p}(x, g(x)) & \leq c(\|x\|) c(0)^{-1}\left\|p_{x}-p(g(x))\right\| \\
& \leq\left\|p_{x}-p(g(x))\right\| \\
& \leq \varphi(x)-\varphi(g(x)) ;
\end{aligned}
$$

so again (*) holds.

Thus Theorem 1 implies $g$ has a fixed point, a contradiction. Hence $\mathrm{y} \in \mathcal{P}(\mathrm{X})$, and $\mathcal{P}$ is surjective.

Now, to show $P$ is open as well, fix $w \in X$ and let $\delta>0$. It suffices to show that $B\left(P_{w} ; \epsilon\right) \subseteq P(B(w ; \delta))$ for a sufficiently small choice of $\epsilon$.

So let $y \in B\left(\mathbb{P}_{w} ; \epsilon\right)$; then $\left\|y-\mathbb{P}_{w}\right\| \leq \epsilon$. Define a mapping $\overline{\mathbf{P}}: B(w ; \delta) \rightarrow Y$ by $\overline{\mathbf{P}}(\mathrm{x})=\mathrm{y}-\mathrm{Px}$. We will show $0 \in \overline{\mathrm{P}}(B(w ; \delta))$, thereby completing the proof of Theorem 2. We accomplish this by applying Theorem 2.1 of [9].

By hypothesis, for $q \in(\mu, 1)$ fixed, there is a $t \in(0,1]$ and an $\bar{x} \in \mathrm{X}$ for which (4) and (5) hold. Hence (4) implies 


$$
\begin{aligned}
& \|\overline{\mathbf{P}} \overline{\mathbf{x}}-(1-t) \overline{\mathrm{P}} \mathbf{x}\|=\|\mathrm{y}-\mathbf{P} \overline{\mathbf{x}}-(1-t)(y-P x)\| \\
& =\|\mathbf{P x}-\mathbf{P} \overline{\mathbf{x}}+t(\mathbf{y}-\mathbf{P} \mathbf{x})\| \\
& \leq q t\left\|y-p_{x}\right\| \\
& =\mathrm{qt}\left\|\overline{\mathrm{p}}_{\mathrm{x}}\right\| \text {, }
\end{aligned}
$$

while (5) implies

$$
\begin{aligned}
\|\bar{x}-\mathbf{x}\| & \leq c(\|\mathbf{x}\|)^{-1} t\|y-\mathbf{p} x\| \\
& =c(\|x\|)^{-1} t\left\|\overline{\mathbf{p}}_{\mathbf{x}}\right\| .
\end{aligned}
$$

Now observe that $\mathrm{x} \in \mathrm{B}(\mathrm{w} ; \delta)$ implies $\|\mathrm{x}-\mathrm{w}\| \leq \delta$. So $\|\mathrm{x}\|-\|\mathrm{w}\| \leq\|\mathrm{x}-\mathrm{w}\|$ yields that $\|x\| \leq \delta+\|w\|$. Applying the function $c$ gives that $c(\delta+\|w\|) \leq$ $c(\|x\|)$. So (17) becomes

$$
\|\bar{x}-x\| \leq c(\delta+\|w\|)^{-1} t\|\overline{\mathbf{p}} \mathbf{x}\| \text {. }
$$

In order to apply Theorem 2.1 of [9] we must verify that

$$
(1-q)^{-1} \int_{0}^{a} s^{-1} B(s) d s \leq \alpha
$$

for appropriate choices of $\alpha, B:[0, \infty) \rightarrow[0, \infty)$ and $a$. If we choose $\alpha=\delta$, $M=c(\delta+\|w\|)^{-1}, B(s)=s$, and $a=c(\delta+\|w\|)^{-1}\left\|\overline{\mathbf{p}}_{\mathrm{w}}\right\| \mathrm{e}^{1-q}$, and if $0<\varepsilon<\delta(1-q) c(\delta+\|w\|) e^{q-1}$, then (19) follows:

$$
\begin{aligned}
(1-q)^{-1} \int_{0}^{a} s^{-1} B(s) d s & =(1-q)^{-1} \int_{0}^{a} d s \\
& =(1-q)^{-1} a \\
& =(1-q)^{-1} c(\delta+\|w\|)^{-1}\left\|\Phi_{1} x\right\| e^{1-q} \\
& \leq(1-q)^{-1} c(\delta+\|w\|)^{-1} \in e^{1-q} \\
& \leq(1-q)^{-1} c(\delta+\|w\|)^{-1} \delta(1-q) \quad c(\delta+\|w\|) e^{q-1} e^{1-q} \\
& =\delta \\
& =\alpha .
\end{aligned}
$$

Hence Theorem 2.1 applies to give the existence of an $x_{0} \in B(w ; \delta)$ for which $\overline{\mathbf{p}}_{\mathrm{x}_{0}}=0$. Thus $\mathrm{y}=\mathrm{Px}_{0}$ and $\mathrm{y} \in \mathcal{P}(\mathrm{b}(\mathrm{x} ; \delta))$, as required.

Theorem 3 is an easy consequence of the Mean Value Theorem.

PROOF. Let $\left\{x_{n}\right\}$ be a sequence in $x$ for which $x_{n} \rightarrow x_{\infty}$ and $P x_{n} \rightarrow y ; 1 . e$. $y=\lim _{n \rightarrow \infty} F x_{n}+6 x_{n}$. Since $\left\{x_{n}\right\}$ is Cauchy, for every $\epsilon>0$ there exists an $N>0$ so that $m, n \geq N$ implies $\left\|x_{n}-x_{m}\right\| \leq \epsilon \mu^{-1} c(0)^{-1}$. Applying the Mean Value Theorem of Mcleod [16] to $G$ yields that

$$
\boldsymbol{G}\left(\mathrm{x}_{\mathrm{n}}\right)-\boldsymbol{G}\left(\mathrm{x}_{\mathrm{m}}\right) \in \overline{\operatorname{co}}\left\{\boldsymbol{G}^{\prime}\left(\mathrm{tx}_{\mathrm{n}}+(1-t) \mathrm{x}_{\mathrm{m}}\right)\left(\mathrm{x}_{\mathrm{n}}-\mathrm{x}_{\mathrm{m}}\right): 0<t<1\right\} ;
$$

from which it follows, via (3), that

$$
\begin{aligned}
\left\|G x_{n}-G x_{m}\right\| & \leq\left\|x_{n}-x_{m}\right\| \sup \left\{\| G^{\prime}\left(t x_{n}+(1-t) x_{m} \|: 0<t<1\right\}\right. \\
& \leq\left\|x_{n}-x_{m}\right\| \sup \left\{\mu c\left(\left\|t x_{n}+(1-t) x_{m}\right\|\right): 0<t<1\right\} \\
& \leq\left\|x_{n}-x_{m}\right\| \mu c(0) \\
& <\epsilon,
\end{aligned}
$$

if $m, n \geq N$.

Hence $\left\{G_{n}\right\}$ is Cauchy in $Y$, so the completeness of $Y$ implies $\left\{\boldsymbol{G x}_{n}\right\}$ converges. By assumption $G$ has closed graph, and so $\lim _{\mathbf{n} \rightarrow \infty} \mathbf{G x}_{n}=\mathbf{G x}_{\infty}$. 
Since $F x_{n}+G x_{n} \rightarrow y$, it follows that $F x_{n} \rightarrow y-G x_{\infty}$. But $F$ has closed graph, so $\mathcal{F x}_{\infty}=\mathrm{y}-\mathcal{G x}_{\infty}$. Therefore $\mathrm{y}=\mathcal{F} \mathrm{x}_{\infty}+\mathcal{G} \mathrm{x}_{\infty}=\mathcal{P} \mathrm{x}_{\infty}$, giving $\mathbf{P}$ closed graph, as desired.

By Theorem 2, $\mathbf{P}$ is an open surjection.

We conclude by remarking that most of our conclusions are fairly direct consequences of earlier results which have used inequalities (4) and (5) as their main assumptions. Thus, for example, surjectivity in Theorem 2 is a special case of Theorem 3.2 in [13], while openness was inferred directly from Theorem 2.1 of [9]. Our main goal here has been to expose a further class of operators to which these more general results apply. The inequalities (4) and (5) have come to play a central role in the theory of normal solvability, and the above results show that these inequalities and the mapping properties they imply are stable under small perturbations.

\section{REFERENCES}

1. POHOZHAYEV, S.I. On the normal solvability of nonlinear operators, Dokl. Akad. Nauk. SSSR 184 (1969), 40-43.

2. BROWDER, F.E. Nonlinear operators and nonlinear equations of evolution in Banach spaces, Proc. Symp. Pure Math. 18, part 3, Amer. Math Soc., Providence, R.I., 1976.

3. BROWDER, F.E. Normal solvability and existence theorems for nonlinear mappings in Banach spaces, Problems in Nonlinear Analysis, Edizioni Cremonese, Roma, Italy, 1971, $\frac{19-35 .}{19}$

4. BROWDER, F.E. Normal solvability for nonlinear mappings and the geometry of Banach spaces, Problems in Nonlinear Analysis, Edizioni Cremonese, Roma, Italy, 1971, 37-66.

5. BROWDER, F.E. Normal solvability for nonlinear mappings in Banach spaces, Bull. Amer. Math. Soc. 77 (1971), 73-77.

6. KIRK, W.A., CARISTI, J. A mapping theorem in metric and Banach spaces, Bul1. Acad. Polon. Sci. Ser. Math. Astronom. Phys. 23 (1975), 891-894.

7. DOWNING, D., KIRK, W.A. A generalization of Caristi's theorem with applications to nonlinear mapping theory, Pacific J. Math. 69 (1977), 339-346.

8. ALTMAN, M. Contractors and Contractor Direction: Theory and Applications, Marcel Dekker, New York, 1977.

9. CRAMER, W. J., RAY, W.O. Solvability of nonlinear operator equations, Pacific J. Math. 95 (1981), 37-50.

10. KOLOMY, J. Normal solvability and solvability of nonlinear equations, Theory of Nonlinear Operators, Akademie Verlag, Berlin, 1974.

11. MOSER, J. A rapidly convergent iteration method and nonlinear differential equations, Ann. Scuola Norm. Sup. Pisa 20 (1966), 499-535.

12. RAY, W.0. A rapidly convergent iteration process and Gateaux differentiable operators, preprint.

13. RAY, W.O., WALKER, A.M. Mapping theorems for Gateaux differentiable and accretive operators, Nonlinear Anal.: TMA 6 (1982), 423-433.

14. RAY, W.0. Normally solvable nonlinear operators, to appear.

15. ROSENHOLTZ, L., RAY, W.O. Mapping theorems for differentiable operators, Bul1. Acad. Polon. Sci. Ser. Sci. Math. 29 (1981), 265-273.

16. MCLEOD, R. Mean value theorems for vector valued functions, Proc. Edinburgh Math. Soc. 14 (1964), 197-209. 


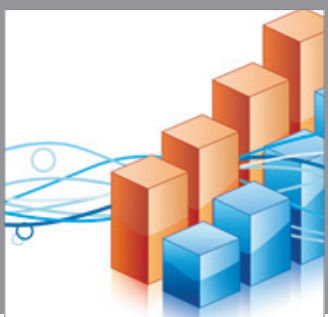

Advances in

Operations Research

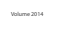

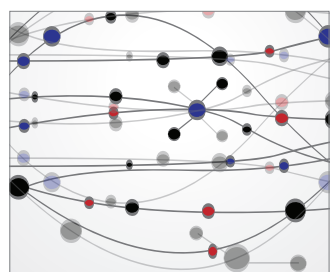

\section{The Scientific} World Journal
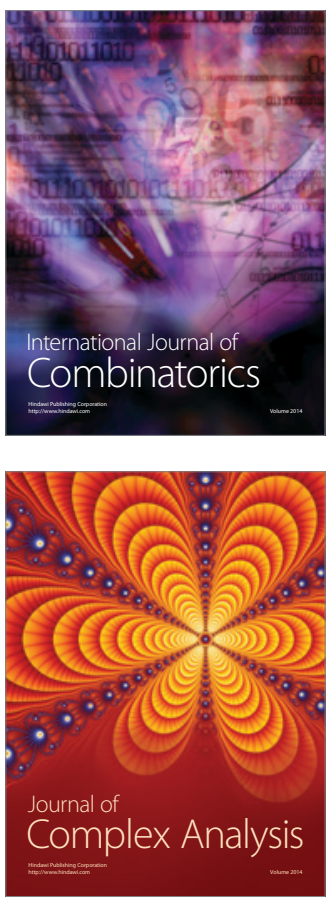

International Journal of

Mathematics and

Mathematical

Sciences
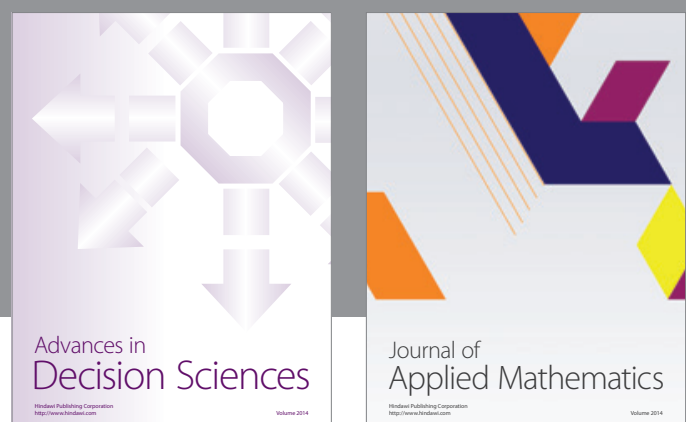

Journal of

Applied Mathematics
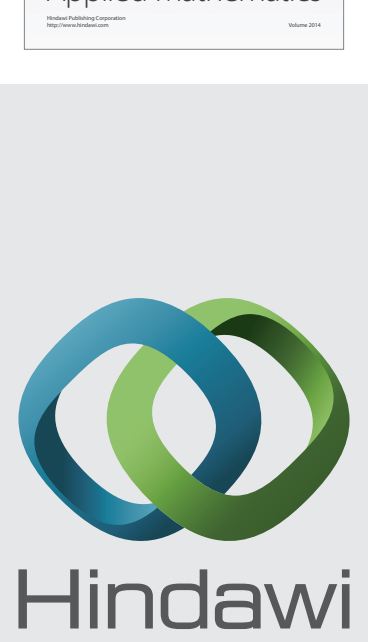

Submit your manuscripts at http://www.hindawi.com
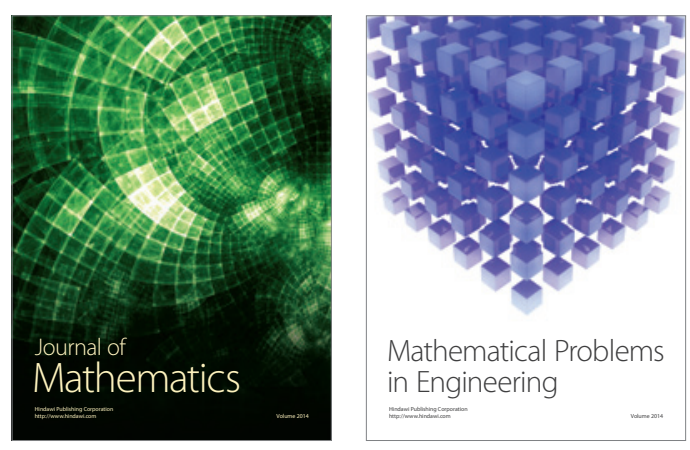

Mathematical Problems in Engineering
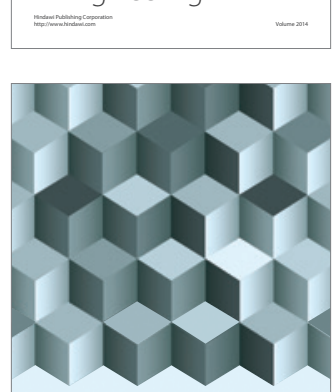

Journal of

Function Spaces
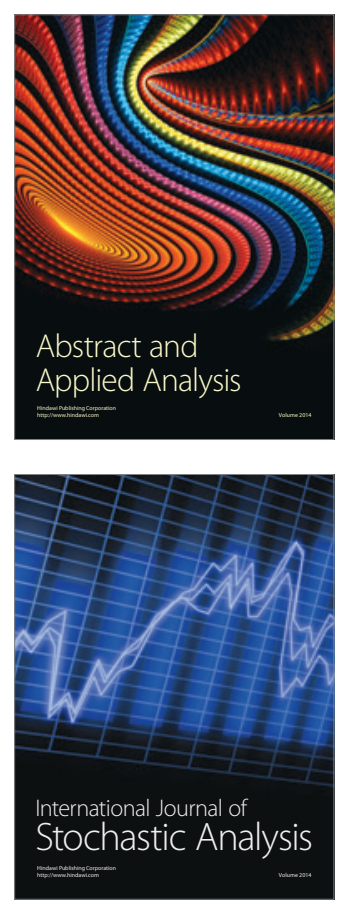

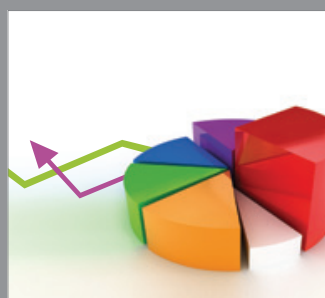

ournal of

Probability and Statistics

Promensencen
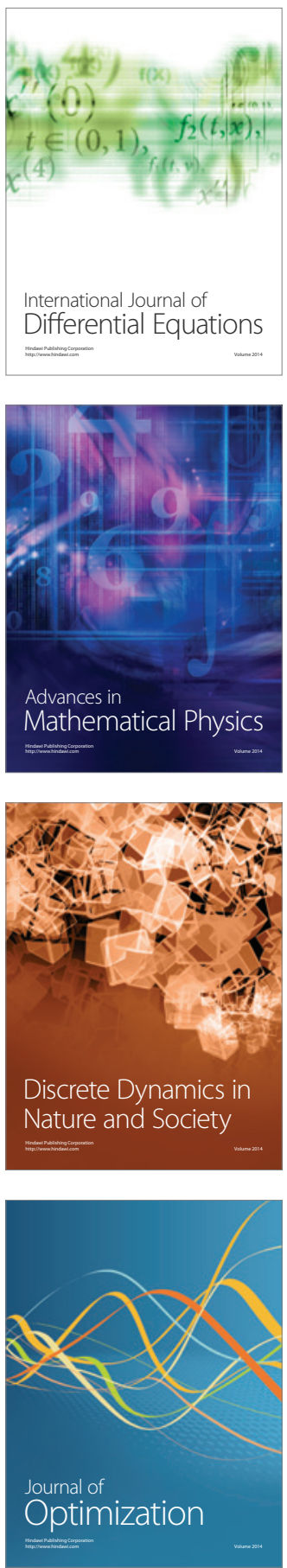